\title{
Article \\ Production of Sustainable Biochemicals by Means of Esterification Reaction and Heterogeneous Acid Catalysts
}

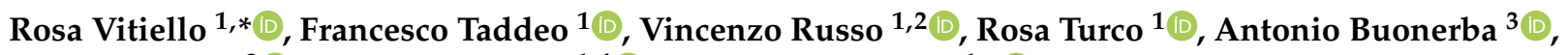 \\ Alfonso Grassi ${ }^{3}{ }^{(\mathbb{D}}$, Martino Di Serio ${ }^{1,4}$ (i) and Riccardo Tesser ${ }^{1, *(\mathbb{B}}$ \\ 1 Department of Chemical Sciences, University of Naples Federico II, 80126 Napoli, Italy; \\ francesco.taddeo@unina.it (F.T.); v.russo@unina.it (V.R.); rosa.turco@unina.it (R.T.); diserio@unina.it (M.D.S.) \\ 2 Laboratory of Industrial Chemistry and Reaction Engineering, Åbo Akademi University, Piispankatu, \\ 20500 Turku, Finland \\ 3 Department of Chemistry and Biology, University of Salerno, 84084 Fisciano, Italy; abuonerba@unisa.it (A.B.); \\ agrassi@unisa.it (A.G.) \\ 4 IROAST, Kumamoto University, Kumamoto 860-8555, Japan \\ * Correspondence: rosa.vitiello@unina.it (R.V.); riccardo.tesser@unina.it (R.T.)
}

\section{check for} updates

Citation: Vitiello, R.; Taddeo, F.; Russo, V.; Turco, R.; Buonerba, A.; Grassi, A.; Di Serio, M.; Tesser, R. Production of Sustainable Biochemicals by Means of Esterification Reaction and Heterogeneous Acid Catalysts. ChemEngineering 2021, 5, 46. https://doi.org/10.3390/ chemengineering5030046

Academic Editor: Luis M. Gandía

Received: 7 July 2021

Accepted: 4 August 2021

Published: 7 August 2021

Publisher's Note: MDPI stays neutral with regard to jurisdictional claims in published maps and institutional affiliations.

Copyright: (c) 2021 by the authors. Licensee MDPI, Basel, Switzerland. This article is an open access article distributed under the terms and conditions of the Creative Commons Attribution (CC BY) license (https:/ / creativecommons.org/licenses/by/ $4.0 /)$.

\begin{abstract}
In recent years, the use of renewable raw materials for the production of chemicals has been the subject of different studies. In particular, the interest of the present study was the use of oleins, mixtures of free fatty acids (FFAs), and oleic acid to produce bio-based components for lubricants formulations and the investigation of the performance of a styrene-divinylbenzene acid resin (sPSB-SA) in the esterification reaction of fatty acids. This resin has shown good activity as a heterogeneous catalyst and high stability at elevated temperatures $\left(180{ }^{\circ} \mathrm{C}\right)$. It was tested in the esterification reaction of oleic acid with 1,3-propanediol and of oleic acid with glycerol. In particular, the esterification reactions were performed in a steel stirred batch reactor and a PBR loop reactor. Tests were conducted varying the reaction conditions, such as alcohol type, temperature, reaction time, and catalysts, both homogeneous and heterogeneous ones. From the obtained results, acid resin (both in reticulated and not-reticulated form) showed high activity in esterification reaction of oleic acid with 1,3-propanediol and of oleic acid with glycerol and good resistance to the deactivation; thus, they can be considered promising candidates for future applications in continuous devices. Viscosity tests were performed, underlining the good properties of the obtained products as lubricant bases.
\end{abstract}

Keywords: oleic acid; biochemicals; esterification; catalysis; lubricants

\section{Introduction}

A lubricant is a substance that reduces friction, providing a protective film between two moving surfaces; thus, lubrication occurs when two surfaces in motion are separated by a lubricant film.

A good lubricant shows characteristics such as high viscosity index, high boiling point, thermal stability, low freezing point, corrosion prevention capability, and high resistance to oxidation [1].

The demand for lubricants in the world was around 35 million tons per year since 1991 with an estimated annual growth of $0.8 \%$ until 2007 and about $2 \%$ since 2012 [1].

Nowadays, most of the used lubricants are still based on mineral oil, although several improvements in lubricant technologies have led to the development of environmentally friendly products in many applications. The replacement of petroleum-based oils with biodegradable products, within lubricants formulation, is the principal way to reduce the negative effects on the ecosystem caused by the intensive use of lubricants [2].

Due to their volatility, petroleum-based lubricants can contaminate the soil and pollute the air, representing a danger for the residents in these areas. Hazardous effects on the respiratory system can be caused by contact with petroleum-based lubricants [3-8]. Vegetable 
products and modified vegetable oil esters can be used as feedstock for the preparation of environmentally friendly and rapidly biodegradable lubricants. For what concerns chemical composition, they are esters of 1,3-propanediol and long-chain fatty acids [9]. However, some of the biodegradable lubricants are based on unmodified vegetable oils; in Europe, for example, rapeseed oil and sunflower oil are predominantly used [9].

Bio-products, such as bio-lubricants, glycerol, or, eventually, bioenergy can be obtained from vegetable oils which are important resources [10-12] but they cannot be directly used as lubricants because of their characteristics, above all, their low oxidative and thermal stability [3]. Nowadays, esters from renewable resources are used in several applications such as lubricants, hydraulic fluids, and greases formulations [13,14]. Important reactions in the field of renewable resources are the transesterification of the glycerol esters or the esterification of the fatty acids [9].

The esterification reaction is a method to reduce the free fatty acids (FFA) content and it is necessary to use these raw materials. The esterification with glycerol is preferably conducted in the presence of homogeneous Lewis acid catalysts, while the research on heterogeneous catalysts is still active.

This way, high FFA conversions are achieved in a shorter time. The product obtained from esterification, in a second step, can be used in transesterification reaction, using an alkaline catalyst.

In the literature [15], glycerol esterification was made mainly in the presence of homogeneous catalysts, considered more active than the heterogeneous ones. In this work, heterogeneous sulfonic resins were used in the esterification of oleic acid with 1,3-propanediol to synthesize lubricant bases.

The advantages of the bio-lubricants derived from waste oils are numerous, such as better lubricity, higher flash point, lower volatility, higher viscosity indices, higher shear stability, higher detergency, and higher resistance to humidity [3,5,11,12,16-25]. Both acidic and basic catalysts can be used for esterification reactions [26]. Among acid catalysts, the most common are p-toluene-sulphonic acid, phosphoric acid, and sulfuric acid, while among basic catalysts, sodium hydroxide, sodium ethoxide, and sodium methoxide are the most used [27]. The problems connected with the use of homogeneous catalysts are the necessity of product purification $[13,14]$ and the reactor corrosion due to liquid catalysts [28-30]. Heterogeneous catalysts show several advantages as they are non-corrosive, environmentally friendly, and are easily separated from the products [31,32]. Among heterogeneous catalysts, Amberlyst 15 is suitable for esterification reaction, but it degrades at $\mathrm{T} \geq 120^{\circ} \mathrm{C}$ [33]. In the esterification reaction, other catalysts such as $\mathrm{H}$ ZSM-5 or H-beta zeolites were also tested [34] but their use is limited due to the small pore size which represents a problem for the reaction with big organic molecules such as FFAs [28,34-38].

Vitiello et al. investigated the performance of $\mathrm{H}_{2} \mathrm{WO}_{4} / \mathrm{SiO}_{2}$ as a new heterogeneous catalytic system characterized by stability at high temperatures in the esterification reaction. In particular, they studied the esterification reaction to obtain a green lubricant from oleic acid and 1,3-propanediol [13,14].

Low-cost raw materials, such as waste oils, have the disadvantage of containing considerable amounts of free fatty acids (FFAs) and water. If this raw material is to be used for the transesterification reaction, this composition has disadvantages in the reaction and in the production process. In particular, the presence of FFA leads to the formation of soaps, which lower the quality of the product and increase the purification costs. Moreover, the presence of water influences the final product quality. In particular, as reported in the literature, small amounts of water shift back the equilibrium of reaction towards the reagents [31]. For this reason, reactions are carried out in such a way that the water that forms during the reaction is continually removed from the reaction environment.

The level of FFAs in waste oil is typically $10-15 \%$ for yellow waste vegetable oil and over $15 \%$ for brown waste vegetable oil [39-41]. 
Starting from the literature review [39], two different esters were synthesized; in particular, in this work, two different reactant systems were considered: (i) oleic acid/1,3propanediol and (ii) oleic acid/glycerol. Regarding the technological applications of this research, the reaction products are known to be lubricating bases [39]. Such synthetic oils include diesters or complex esters as good base lubricants; various conventional additives may be added to the compositions including oxidation inhibitors, rust preventives, and other thickeners to improve lubricant properties.

\section{Materials and Methods}

\subsection{Materials}

A commercial acid resin, Amberlyst-15, and two non-commercial resins, multiblock sulfonated syndiotactic-co-1,4-cis-polybutadiene copolymers (sPSB-SA) catalysts were used in the esterification reaction. In detail, they were used as acid resins sPSB-SA1, non-crosslinked sulfonic resin, and sPSB-SA2, the same crosslinked sulphonic resin. All reagents used for reactions and procedures were purchased from Merck at the maximum purity level available and were used without further purification.

\section{2. sPSB Sulfonation and Characterization}

As reported in previous papers [42,43] multiblock copolymers syndiotactic polystyreneco-1,4-cis-polybutadiene (sPSB) was synthesized. sPSB resin was sulfonated according to the procedure reported in a previous paper [42]. Two resins (reticulated and nonreticulated) were tested in the esterification reaction. These resins are copolymers made by $55 \mathrm{~mol} \%$ of butadiene. These copolymers are made by segments of polystyrene and predominantly 1,4-cis-polybutadiene (molar ratio 1,4-cis-polybutadiene/1,2-polybutadiene equal to 0.85 ). The polystyrene fraction gives the polymer high thermal, mechanical, and chemical resistance and makes it a suitable substrate for the synthesis of sulfonated polymers. The characterization (chemical and structural) of these resins was already reported in previous work [42]. The most important characteristic of the resins reported in this work is their excellent thermal stability. In particular, the resins used in this work as catalysts in the esterification reaction, despite having a structure similar to that of commercial sulfonic resins, retain their structure, even at temperatures above $120^{\circ} \mathrm{C}$ (up to $200{ }^{\circ} \mathrm{C}$ ).

\subsection{Free Fatty Acid (FFA) Esterification}

A carboxylic acid, oleic acid, to simulate environmentally friendly feedstock oil and alcohol, 1,3-propanediol and glycerol, were esterified in a steel stirred batch reactor. On the reactor head, a nitrogen stripping flow was installed to remove the water formed during the reaction. In this way, it was possible to continuously remove water formed during the reaction to shift the equilibrium towards reaction products.

On the other part of the reactor head, a thermocouple was placed to control the reaction temperature. In this reactor configuration, the heterogeneous catalyst is dispersed in the liquid, and it must be separated from the reaction mixture at the end of the reaction.

One of the two reactants was loaded firstly in the reactor and was heated up to the desired temperature; when it was reached, the other reactant and the catalyst were loaded. This is considered the beginning of the reaction. The scheme of this reactor is reported in Figure 1.

Another configuration used to carry out esterification tests is the PBR loop reactor (Figure 2). This reaction system consists of a stirred jacketed vessel having a volume of $1 \mathrm{~L}$ connected from the bottom through a pump to a $0.1 \mathrm{~L}$ packed bed reactor which is connected back from the top to the batch reactor. The temperature of the batch reactor is regulated using a thermostatted oil bath and it is controlled by a thermocouple placed inside the reactor itself. The temperature of the tubular reactor, instead, is regulated by a thermoregulator through a heating jacket and it is controlled by a thermocouple placed in the upper part of the reactor. The system is connected to a nitrogen cylinder and a flowmeter that regulates the gas flow. 


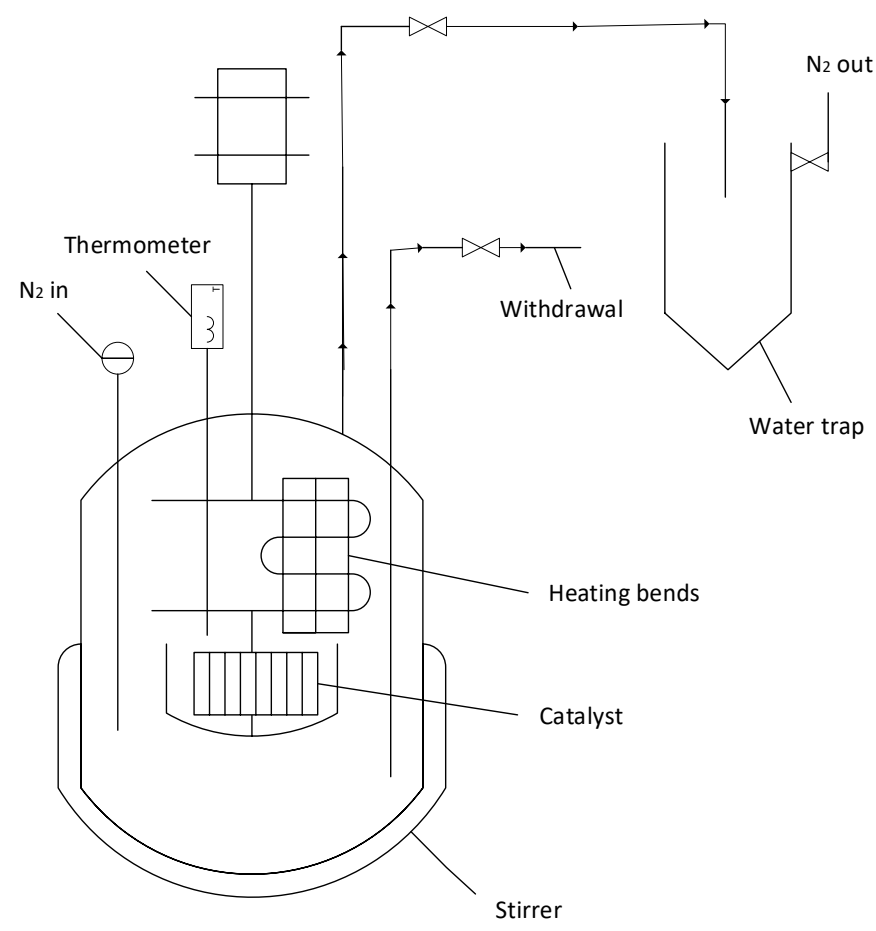

Figure 1. Batch reactor configuration.

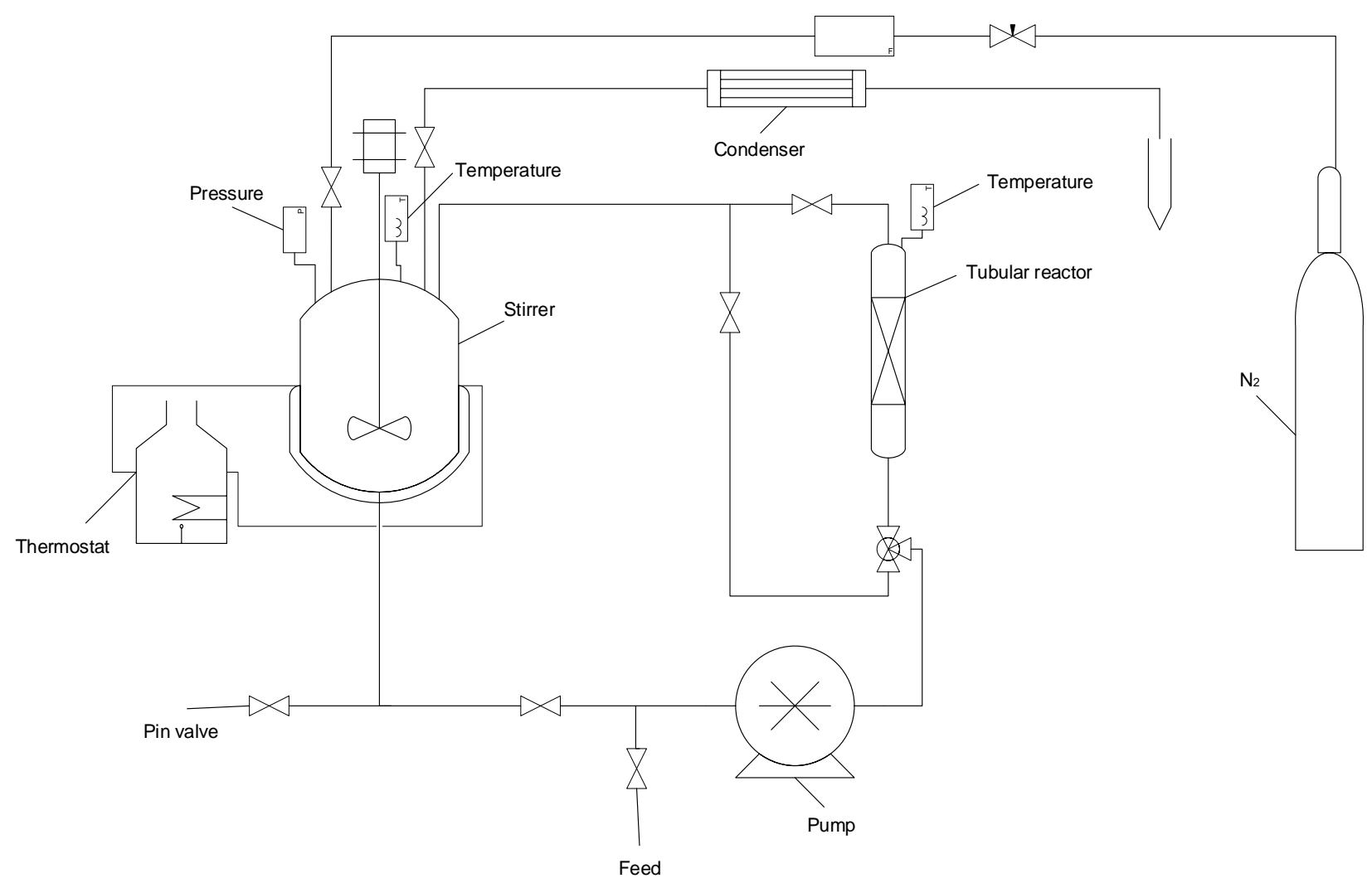

Figure 2. PBR loop reactor configuration.

With this configuration, there is a separation between the reaction zone, the tubular reactor in which the catalyst is loaded, and a stripping zone (the vessel) where the water formed during the esterification reaction is removed using the nitrogen flow and a condenser placed on the upper part of the reactor. 
Reagents are fed to the batch reactor through a bypass line to avoid flowing through the reactor itself before the beginning of the reaction due to the presence of the catalyst.

They are mixed in the vessel and flow through the tubular reactor where the reaction occurs. This system is discontinuous, and it permits to operate at high flows. In this way, even if the per-pass conversion degrees are low, the partially converted fluid flows several times through the catalyst bed, obtaining a high conversion degree at the end of the reaction.

The advantage of this configuration is that the catalyst is confined in the tubular reactor, so, with this reaction system, the catalyst filtration step is eliminated; therefore, products do not need to be separated from the catalyst itself at the end of the reaction, simplifying the possible industrial process. Moreover, by imposing high recirculation flows, fluid-solid mass transfer resistance would be surely limited, allowing to work in kinetic regime [13].

\subsubsection{Catalyst Liquid Take-Up}

Using a heterogeneous catalyst, such as an ionic resin, an important phenomenon is its liquid take-up capacity due to the interaction with the reaction mixtures and the catalyst's tendency to include organic molecules within its structure, leading to an increase in the catalyst dimension. Depending on the resin chemical nature, some of them are porous enough to allow reaction mixture access without swelling; others must swell to accommodate large molecules. The amount of swelling is determined by placing dry resin in a graduated cylinder and adding an excess of solvents. Its volume is measured before the addition of the reaction mixture and when the resin has finished swelling [44].

In this experimental test, the catalyst was placed in an excess of reagent so that the swelling phenomenon occurred; after that, its volume increased, and it was loaded in the tubular reactor.

In particular, three different feeds were tested for the swelling phenomena: alcohol, acid, and a reaction mixture (alcohol + acid).

\subsubsection{Analytical Methods}

The analytical method used to investigate the progress of the reaction and the conversion of the carboxylic acid follows the legislation ASTM D974 [45], which consists of acid-base titrations to determine the residual acidity (acidity number) and subsequently the conversion of carboxylic acid. The sample was titrated with a $\mathrm{KOH}$ solution of a known concentration of $0.1 \mathrm{M}$. As reported in the legislation, ethanol was used as a solvent to dissolve the sample and alcoholic phenolphthalein as an indicator. In detail, the titration was performed weighing $0.30 \mathrm{~g}$ of sample dissolved in $20 \mathrm{~mL}$ of ethanol. The acidity number, which represents the acidity of a carboxylic acid, is defined as the amount of potassium hydroxide, expressed as $\mathrm{mg}$ of $\mathrm{KOH}$ needed to neutralize one gram of sample. It is evaluated through the following Equation (1):

$$
N . A .=\frac{\left(V-V_{0}\right) \cdot C \cdot M_{W}}{W}
$$

where:

N.A.: acidity number (mg $\mathrm{KOH} / \mathrm{g}$ of the sample)

$V$ : volume of solution used in the titration $(\mathrm{mL})$

$V_{0}$ : volume of solution used in the titration of blank (ethanol) $(\mathrm{mL})$

C: titrant solution concentration $(\mathrm{mol} / \mathrm{L})$

$M_{W}$ : titrant molecular weight $(\mathrm{g} / \mathrm{mol})$

W: sample mass (g)

Carboxylic acid conversion $\left(x_{c a}\right)$ is calculated through Equation (2):

$$
x_{c a}=\frac{N A_{0}-N A_{t}}{N A_{0}}
$$


where:

$N A_{0}$ : initial mixture acidity number

$N A_{t}$ : mixture acidity number at time $t$.

As we are interested in the reaction progress, in the experimental data tables, only carboxylic acid conversion is reported, and no acidity number.

\subsubsection{Viscosity Test}

The viscosity tests were made at two typical temperatures $\left(40{ }^{\circ} \mathrm{C}\right.$ and $\left.100{ }^{\circ} \mathrm{C}\right)$ using the rheometer Anton Paar series MCR 92 equipped with a Peltier system automatic temperature control. Tests were performed on the lubricant bases themselves without adding any solvents.

\subsubsection{Experimental Tests}

Tests were carried out in a batch reactor (see Figure 1) firstly without catalyst and then using homogeneous and heterogeneous catalysts.

Different tests were made varying the reaction conditions, i.e., alcohols, temperature, reaction time, and catalysts. In the following tables, details are reported. Blank tests (without catalyst) were performed at different temperatures, as reported in Table 1 . The reactions were carried out using a stoichiometric ratio on the functional groups between alcoholic groups and acid groups. In particular, considering the first system oleic acid and 1,3-propanediol, the reactions were carried out using two moles of acid per mole of alcohol, considering that oleic acid had one acid group and 1,3-propanediol had two alcoholic functions. For the second reaction system, instead, tests were performed using three moles of oleic acid with one mole of glycerol. In these conditions, the same molar ratio was always used considering the functional groups.

Table 1. Blank tests (acid: oleic acid, times: $180 \mathrm{~min}$ ).

\begin{tabular}{ccc}
\hline Run & Alcohol & $\boldsymbol{T}\left[{ }^{\circ} \mathbf{C}\right]$ \\
\hline $\mathbf{1}$ & 1,3-propanediol & 120 \\
$\mathbf{2}$ & 1,3-propanediol & 150 \\
$\mathbf{3}$ & 1,3-propanediol & 180 \\
$\mathbf{4}$ & 1,3-propanediol & 200 \\
$\mathbf{5}$ & Glycerol & 120 \\
$\mathbf{6}$ & Glycerol & 150 \\
$\mathbf{7}$ & Glycerol & 180 \\
$\mathbf{8}$ & Glycerol & 200 \\
\hline
\end{tabular}

Different types of heterogeneous catalyst were used in the esterification reaction; resin properties are reported in Table 2.

Table 2. Properties of used resins.

\begin{tabular}{ccc}
\hline Resin & Acidity $\left[\mathbf{m e q H}{ }^{+} / \mathbf{g}\right]$ & T max \\
{$\left[{ }^{\circ} \mathbf{C}\right]$}
\end{tabular}

In Tables 3-5, catalyzed tests are reported. A comparison was made between tests performed in the presence of sulfuric acid as a homogeneous catalyst and sulfonic resins as heterogeneous catalysts. 
Table 3. Catalyzed tests using molar alcohol/acid $=1 / 2$ for 1-3, propanediol/oleic acid and molar alcohol/acid $=1 / 3$ for glycerol/oleic acid, catalyst $1 \mathrm{wt} \%$ vs. acid content, temperature $=120^{\circ} \mathrm{C}$ and time $=180 \mathrm{~min}$.

\begin{tabular}{cccc}
\hline Run & Alcohol & Acid & Catalyst \\
\hline $\mathbf{9}$ & 1,3-propanediol & Oleic acid & Amberlyst 15 \\
$\mathbf{1 0}$ & 1,3-propanediol & Oleic acid & Nafion R50 \\
$\mathbf{1 1}$ & 1,3-propanediol & Oleic acid & Sulfonic reticulated resin \\
$\mathbf{1 2}$ & 1,3-propanediol & Oleic acid & Sulfonic non-reticulated resin \\
$\mathbf{1 3}$ & 1,3-propanediol & Oleic acid & $\mathrm{H}_{2} \mathrm{SO}_{4}$ \\
$\mathbf{1 4}$ & Glycerol & Oleic acid & Amberlyst 15 \\
$\mathbf{1 5}$ & Glycerol & Oleic acid & Nafion R50 \\
$\mathbf{1 6}$ & Glycerol & Oleic acid & Sulfonic reticulated resin \\
$\mathbf{1 7}$ & Glycerol & Oleic acid & Sulfonic non-reticulated resin \\
$\mathbf{1 8}$ & Glycerol & Oleic acid & $\mathrm{H}_{2} \mathrm{SO}_{4}$ \\
\hline
\end{tabular}

Table 4. Catalyzed tests using oleic acid, molar ratio alcohol $/$ acid $=1 / 2$, temperature $=180{ }^{\circ} \mathrm{C}$.

\begin{tabular}{cccc}
\hline Run & Alcohol & Catalyst & Time [h] \\
\hline $\mathbf{1 9}$ & 1,3-propanediol & $\mathrm{H}_{2} \mathrm{SO}_{4}$ & 3 \\
$\mathbf{2 0}$ & 1,3-propanediol & Non-reticulated resin & 3 \\
$\mathbf{2 1}$ & 1,3-propanediol & Reticulated resin & 3 \\
$\mathbf{2 2}$ & 1,3-propanediol & $\mathrm{H}_{2} \mathrm{SO}_{4}$ & 6 \\
$\mathbf{2 3}$ & 1,3-propanediol & Non-reticulated resin & 6 \\
$\mathbf{2 4}$ & 1,3-propanediol & Reticulated resin & 6 \\
\hline
\end{tabular}

Table 5. Catalyzed tests using oleic acid, molar ratio alcohol $/$ acid $=1 / 3$, temperature $=180^{\circ} \mathrm{C}$.

\begin{tabular}{cccc}
\hline Run & Alcohol & Catalyst & Time [h] \\
\hline $\mathbf{2 5}$ & Glycerol & $\mathrm{H}_{2} \mathrm{SO}_{4}$ & 3 \\
$\mathbf{2 6}$ & Glycerol & Non-reticulated resin & 3 \\
$\mathbf{2 7}$ & Glycerol & Reticulated resin & 3 \\
$\mathbf{2 8}$ & Glycerol & Non-reticulated resin & 6 \\
$\mathbf{2 9}$ & Glycerol & Non-reticulated resin & 6 \\
$\mathbf{3 0}$ & Glycerol & $\mathrm{H}_{2} \mathrm{SO}_{4}$ & 6 \\
\hline
\end{tabular}

All tests reported in the table were performed using a batch reactor configuration. First of all, the commercial heterogeneous catalysts were tested, and also synthetic heterogeneous catalysts working at $120{ }^{\circ} \mathrm{C}$, the maximum temperature to work using Amberlyst 15 to verify the activity at this temperature.

In a second phase of the work, higher temperature concerning the previous runs, $180{ }^{\circ} \mathrm{C}$, was adopted. At this temperature, synthetic heterogeneous catalysts, reticulated and not reticulated, in comparison with homogeneous catalysts, were tested in a batch reactor configuration. Heterogeneous catalysts were tested both for the reactive system 1,3-propanediol/oleic acid and glycerol/oleic acid. The runs were carried out to identify the best synthetic catalyst in terms of activity concerning the acid groups. Starting from the results obtained using a batch configuration reactor, the stability of the best catalyst, in terms of activity was studied using a loop reactor configuration. All tests were compared with the tests carried out without catalyst.

\section{Results}

\subsection{Catalyst Take-Up Results}

The results obtained from the swelling test have demonstrated that this phenomenon can be ignored for reticulated resin, but it is significant in the case of the non-reticulated one. It was observed that for the alcohol test and alcohol + acid test, it is possible to increase the resin volume by $30 \%$. This aspect is important when we consider the recycle loop reactor 
because, in this case, the catalyst is loaded in a tubular reactor with a defined volume to avoid the formation of blockages.

\subsection{Blank Tests}

The obtained results in the tests made without a catalyst for the system 1,3-propanediol/ oleic acid and glycerol/oleic acid (conditions reported in Table 1) are reported, respectively, in Figure 3a runs 1-4, and in Figure $3 b$ runs 5-8, respectively.
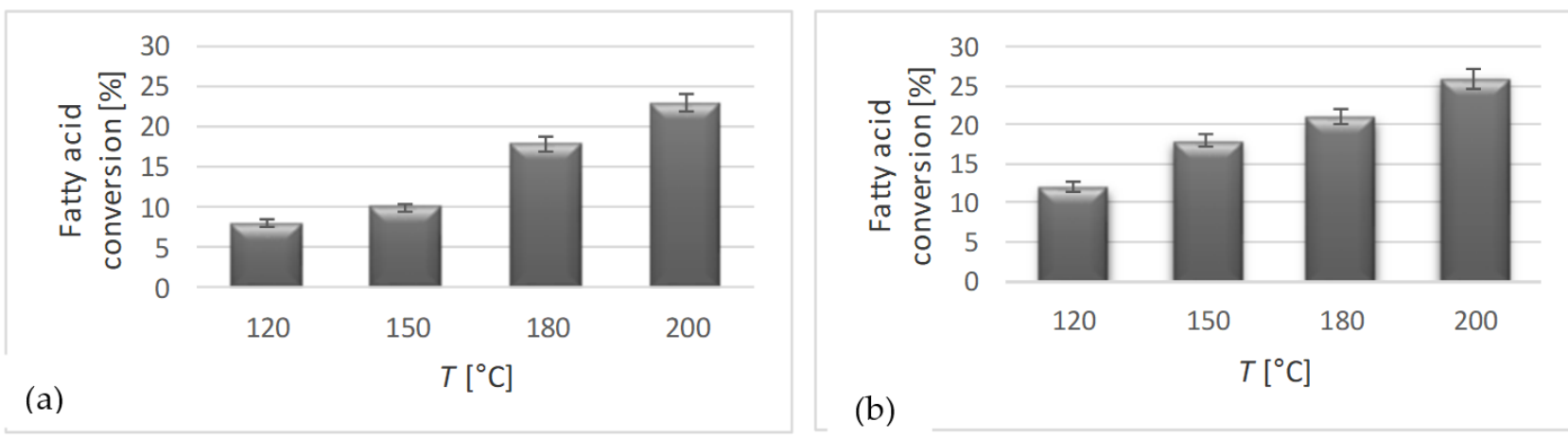

Figure 3. (a) Oleic acid/1,3-propanediol blank tests, runs 1-4, (b) oleic acid/glycerol blank tests, runs 5-8.

As can be seen in Figure 3, the maximum reached conversion degree is $18 \%$ at $180^{\circ} \mathrm{C}$ for the system oleic acid/1,3-propanediol (run1), while it is $21 \%$ at $180{ }^{\circ} \mathrm{C}$ (run 7) for the system oleic acid/glycerol. At $200{ }^{\circ} \mathrm{C}$ for the system with 1,3-propanediol (run 2), it is possible to observe a conversion value of $23 \%$; for the system with glycerol (run 8 ), the conversion value is $26 \%$. From these results, it is possible to note that there is a significant blank contribution. Moreover, conversions obtained with glycerol are always slightly higher than the ones reached with 1,3-propanediol, showing a higher activity of the triol compared to the diol.

\subsection{Catalytic Tests}

Different catalysts were tested in the oleic acid esterification with 1,3-propanediol (Figure 4a) and glycerol (Figure 4b).

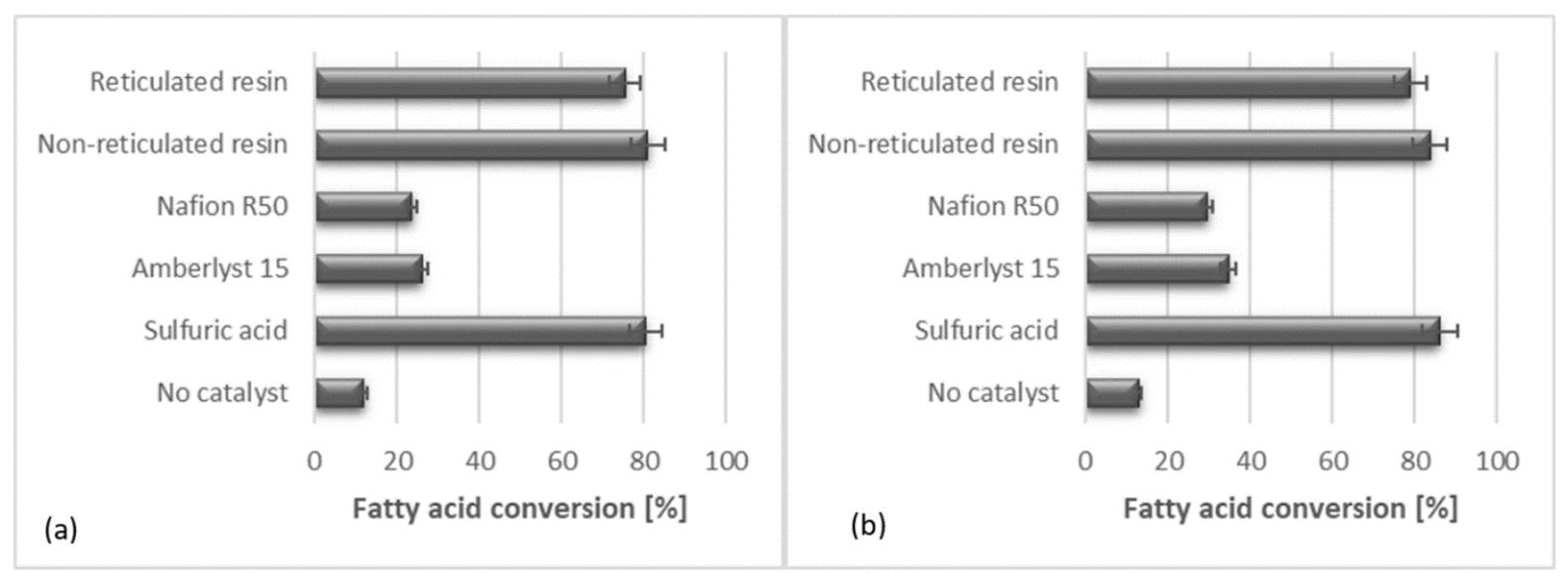

Figure 4. (a) Operative conditions: 1,3-propanediol/oleic acid $=1 / 2$; catalyst amount $=1.8 \times 10^{-3} \mathrm{meqH}^{+} ; \mathrm{T}=120^{\circ} \mathrm{C}$; time $=180 \mathrm{~min} .(\mathbf{b})$ Operative conditions: glycerol/oleic acid $=1 / 3$; catalyst amount $=1.8 \times 10^{-3} \mathrm{meqH}^{+} ; \mathrm{T}^{-120}{ }^{\circ} \mathrm{C}$; time $=180 \mathrm{~min}$.

Both reticulated and not-reticulated resins can resist high temperatures up to $200^{\circ} \mathrm{C}$ but they have different acidity: the non-reticulated resin has an acidity equal to $5.4 \mathrm{meqH}^{+} / \mathrm{g}$, while the acidity of the reticulated resin is equal to $4.3 \mathrm{meqH}^{+} / \mathrm{g}$ [42]. 
The reticulated resin shows a lower acidity, but it shows higher stability with the possibility to reuse the resin itself for subsequent reaction cycles due to its resistance to deactivation.

It is possible to observe for the system oleic acid/1,3-propanediol (Figure 4a) that the highest conversion values are achieved using sulfonic resins and these values are similar to those obtained using sulfuric acid. In this case, it is possible to note that to work with the same equivalent of acid group, of homogeneous and heterogeneous catalysts, the same results were obtained in terms of activity at $120{ }^{\circ} \mathrm{C}$. At this temperature, the blank contribution to the conversion is low (around 8\%) but the values obtained with the use of sulfonic resins and sulfuric acid is high; using sulfuric acid, 80\% was reached, compared with $81 \%$ for non-reticulated resin and $75 \%$ with reticulated ones, respectively.

In their study, Maquirriain et al. [15] showed that in the glycerol esterification, homogeneous catalysts (e.g., sulfuric acid, p-toluenesulfonic acid, and methanesulfonic acid) were preferred to the heterogeneous ones thanks to their higher activity. In our work, it was seen that for the system oleic acid-glycerol (Figure $4 \mathrm{~b}$ ) the highest conversion values are achieved using sulfonic resins and these values are comparable to those obtained using sulfuric acid. At $120{ }^{\circ} \mathrm{C}$, the blank contribution to the conversion, in this case, is also low $(13 \%)$, but the values obtained with the use of sulfonic resins and sulfuric acid is high; using sulfuric acid, $86 \%$ was reached, with non-reticulated resin, $83 \%$, and with reticulated resins, $78 \%$, respectively.

Considering the good results obtained using sulfonic resins (both reticulated and nonreticulated), the reaction was carried out using higher temperatures. The most important difference between commercial resins and sulfonic resins used in this work is the stability at a temperature higher than $120{ }^{\circ} \mathrm{C}$. In particular, Amberlyst 15 tested in this work as a reference cannot be used at a temperature higher than $120^{\circ} \mathrm{C}$. Nafion R50, instead, can be used up to a temperature of $190^{\circ} \mathrm{C}$ but it is not stable at this temperature. For this reason, the performances of sulfuric resins at $180^{\circ} \mathrm{C}$ were studied in comparison with tests using sulfuric acid as a homogeneous reference.

In Figure 5, tests with oleic acid and 1,3-propanediol without catalyst, with sulfuric acid as a homogeneous reference, with sulfonic reticulated and non-reticulated resin as heterogeneous catalysts are reported. These experiments were performed at $180{ }^{\circ} \mathrm{C}$. It is possible to see that after $150 \mathrm{~min}$, conversions obtained with reticulated and non-reticulated sulfonic resins were comparable to that of sulfuric acid.

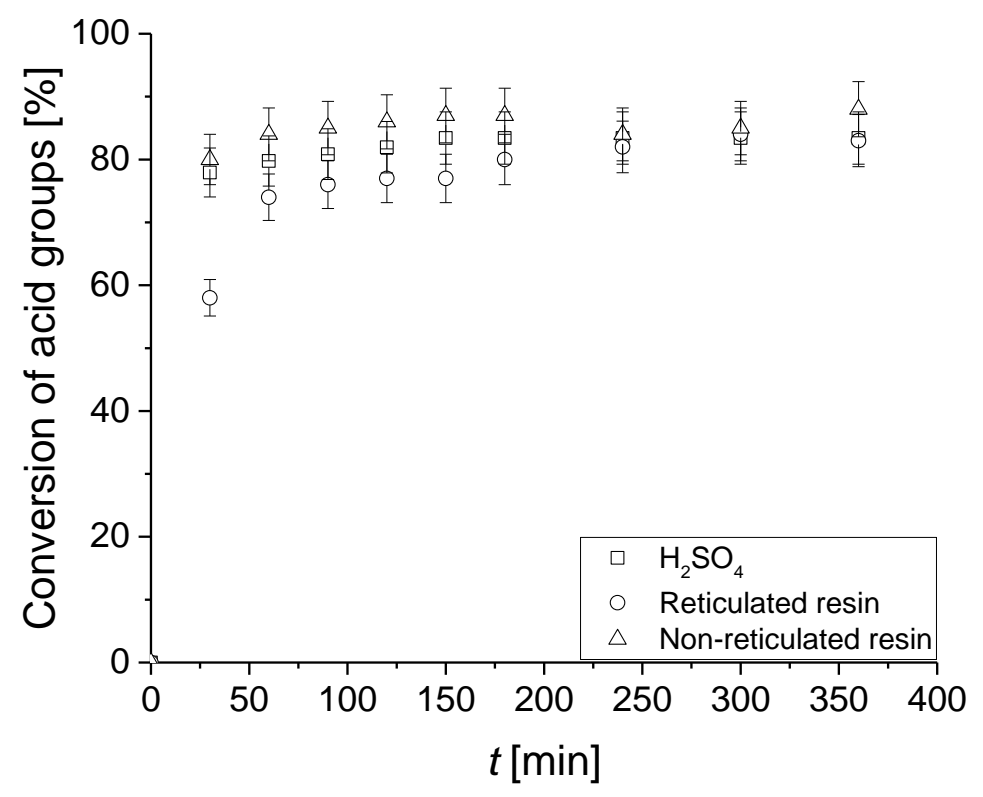

Figure 5. Operative conditions: molar ratio 1,3-propanediol/oleic acid $=1 / 2$; catalyst amount $=1.8 \times 10^{-3} \mathrm{meqH}^{+} ; \mathrm{T}=180^{\circ} \mathrm{C}$; time $=360 \mathrm{~min}$. 
The activity of reticulated resin was always slightly lower than non-reticulated resin also if we worked using the same amount (in equivalent) of acid groups. This can be due to the different distribution of acid groups on the surface of catalysts and, probably, for the different swelling ratios of the two catalysts [31,42]. The reticulated resin has a more rigid structure and lower swelling and accessibility of reactants inside the particles. The difference in activity should be considered as not highly significant, and if we observe the error bars in Figure 4, the behavior of the two resins is quite equivalent.

Some experiments, similar to those reported in Figure 5 for the reactant system oleic acid-1,3-propanediol, were carried out also for the reactant system oleic acid-glycerol. The results obtained for this second system followed the same trend of the first reactant system results, as can be seen in the following figure (Figure 6).

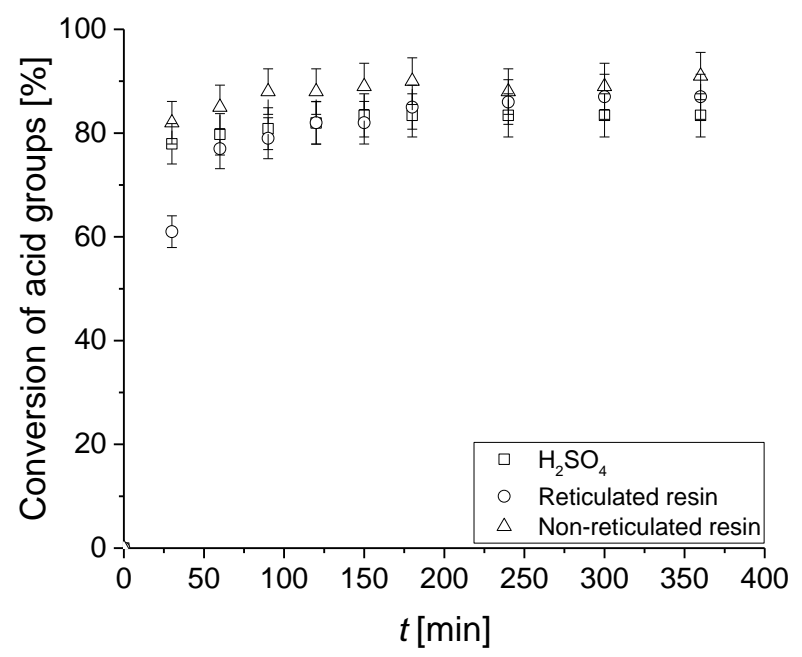

Figure 6. Operative conditions: molar ratio glycerol/oleic acid $=1 / 3$; catalyst amount $=1.8 \times 10^{-3} \mathrm{meqH}^{+} ; T=180^{\circ} \mathrm{C}$; time $=360 \mathrm{~min}$.

From the obtained results, it is possible to conclude that the sulfonic resins are good catalysts in terms of activity, also in comparison with sulfuric acid. For this reason, it was decided to also investigate the aspect of their stability. In particular, tests were performed using the reactant system oleic acid/1,3 propanediol.

\subsection{Reusability Test of the Catalysts in the PBR Loop Reactor}

When using heterogeneous catalysts, another important insight is related to its reusability during long-term use. Different types of reactors have been proposed in the literature [46,47], but in this work, the life of catalysts was studied using a tubular reactor in loop configuration, as described in a previous section. The tests were carried out by loading the loop reactor with the catalyst, reticulated and non-reticulated, and changing only the reagents with fresh ones after the reaction step. The results of this set of experiments are reported in

It is possible to observe that the activity of the non-reticulated resin slightly decreases during its successive reuses, while in the case of the reticulated resin, the activity remains quite constant. A sequence of 10 successive reaction steps has been conducted with this modality with the same amount of catalyst initially charged.

This reactor configuration is intermediate between batch and continuous reactors. It makes the operations very similar to a continuous reactor.

An important result obtained with the loop reactor is the possibility to use the same catalyst for many reaction cycles. In this work, the same catalyst was used 10 times, obtaining similar results, so it is expected that it can be used for other tests without losing activity. This aspect can represent an advantage both from an economic and practical point of view, especially for a possible industrial scale-up. Furthermore, as previously mentioned, 
since the catalyst is loaded within the tubular reactor, product recovery operations are simplified due to the absence of the catalyst filtration step. Figure 7.

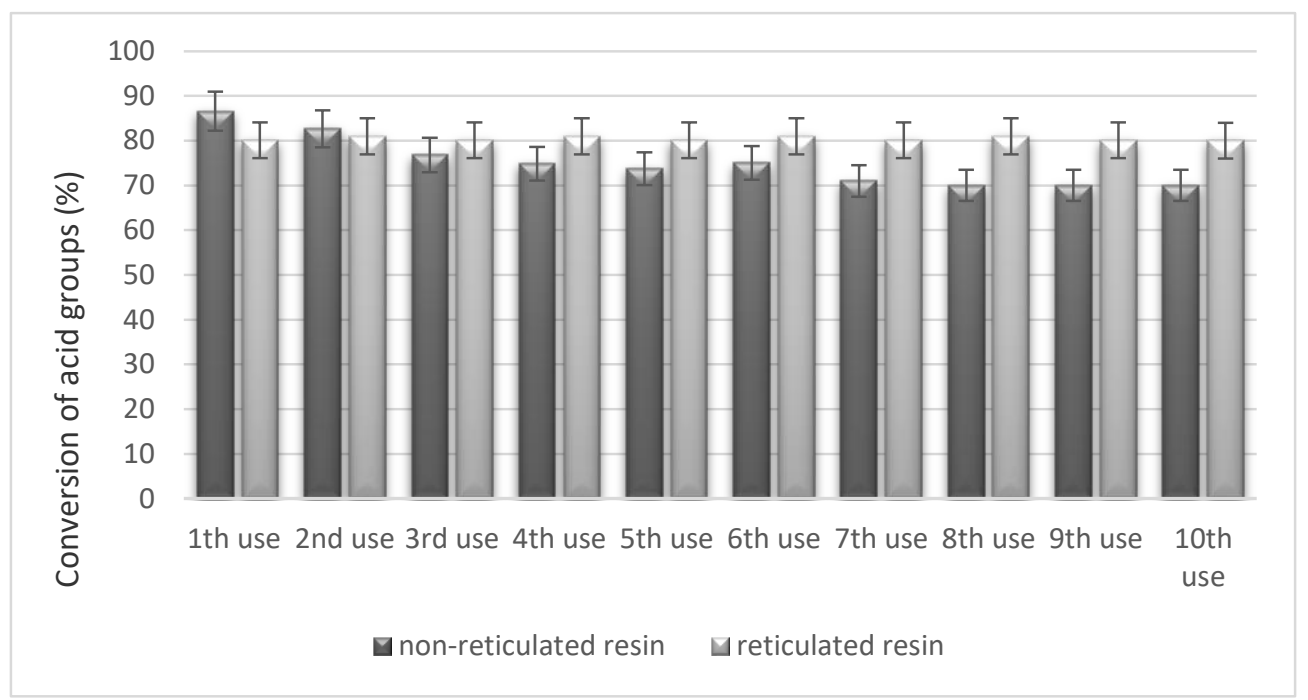

Figure 7. Operative conditions: oleic acid/1,3-propanediol $=1$ considering the functional groups; catalyst amount $=1.8 \times 10^{-3} \mathrm{meqH}^{+} ; \mathrm{T}=180^{\circ} \mathrm{C}$; time $=180 \mathrm{~min}$.

The reactive system glycerol/oleic acid was tested in a loop reactor only with reticulated resin that showed a stable activity during the ten successive runs. However, in this system, the final product showed high viscosity; consequently, we observed a high pressure drop across the packed bed tubular reactor.

\subsection{Viscosity Tests}

The synthesized products from the esterification of oleic acid with 1,3-propanediol were subjected to a viscosity test to compare their viscosity with the viscosity of common lubricant base oils. The resulted values for all the products are reported in Table 6. The product (synthetic base lubricant) used in the viscosity test is a typic product derived from the esterification reaction in the presence of the non-reticulated sulfonic resin as the catalyst, which allowed it to reach the highest conversion degree.

Table 6. Viscosity tests results.

\begin{tabular}{ccc}
\hline Product & Viscosity at $\mathbf{4 0}{ }^{\circ} \mathbf{C}[\mathbf{m P a} \cdot \mathbf{s}]$ & Viscosity at $\mathbf{1 0 0}{ }^{\circ} \mathbf{C}[\mathbf{m P a} \cdot \mathbf{s}]$ \\
\hline Synthetic base lubricant & 25.1 & 7.2 \\
80 N lubricant base oil & 12.0 & 3.2 \\
150 N lubricant base oil & 29.6 & 5.4 \\
\hline
\end{tabular}

As can be seen from Table 6, the analyzed product (synthetic base lubricant) showed a viscosity value much greater than an $80 \mathrm{~N}$ base oil and very similar to a $150 \mathrm{~N}$ base oil. The obtained results highlight that in the esterification reaction a higher conversion degree was reached with the sulfonic resins as the catalyst. Furthermore, the obtained products showed good properties as lubricant bases, as evidenced by the viscosity tests.

\section{Conclusions}

In the present paper, the esterification of oleic acid with a diol (1,3-propanediol) was conducted, to produce bio-lubricant bases. Different catalysts were tested to check both their activity and reusability in the perspective of an industrial application.

Both reticulated and non-reticulated resins showed good activity in catalyzing the esterification of oleic acid, independently of the choice of the alcohol. 
The good results obtained for stability tests with reticulated resin led us to conclude that for the esterification reaction, to produce lubricant bases, the reticulated resin shows the best performance; even with an activity slightly less than non-reticulated resin, it had more stability over time, and this could probably represent the best compromise. This information is of the outermost importance, as a real industrial application requires a stable catalyst in the long run. The obtained products showed a viscosity greater than a common $80 \mathrm{~N}$ base oil and comparable with the viscosity of a $150 \mathrm{~N}$ lubricant base oil. Taking as a reference the work conducted by Maquirriain et al. [15], in which the authors used homogeneous catalysts, in our work, glycerol was also used as polyalcohol to confirm the good activity of the sulfonic resins in the esterification reaction. Additionally, in this case, the results showed an excellent activity of the synthesized resin.

In perspective, this result can be considered as the starting point of a deeper work dedicated to the scale-up operation from batch to continuous application, to improve the productivity of the system.

Author Contributions: Conceptualization, R.V. and R.T. (Riccardo Tesser); methodology, R.T. (Rosa Turco); software, V.R.; validation, M.D.S.; formal analysis, R.T. (Riccardo Tesser); investigation, F.T., V.R., A.B. and A.G.; resources, M.D.S.; data curation, F.T.; writing — original draft preparation, R.V.; writing-review and editing, R.V. and R.T. (Riccardo Tesser); visualization, M.D.S.; supervision, R.T. (Riccardo Tesser); project administration M.D.S.; funding acquistion, M.D.S. All authors have read and agreed to the published version of the manuscript.

Funding: This research received no external funding.

Institutional Review Board Statement: Not applicable.

Data Availability Statement: Not applicable.

Acknowledgments: The authors wish to thank the European Union (FSE, PON Ricerca e Innovazione 2014-2020, Azione I.1 “Dottorati Innovativi con caratterizzazione Industriale”) for funding a Ph.D. grant to Francesco Taddeo.

Conflicts of Interest: The authors declare that there are no conflict of interest.

\section{References}

1. Mobarak, H.M.; Niza Mohamad, E.; Masjuki, H.H.; Kalam, M.A.; Al Mahmud, K.A.H.; Habibullah, M.; Ashraful, A.M. The prospects of biolubricants as alternatives in auto motive applications. Renew. Sustain. Energy Rev. 2014, 33, 34-43. [CrossRef]

2. Bart, J.C.J.; Gucciardi, E.; Cavallaro, S. Biolubricant: Science and Technology; Woodhead Publishing: Sawston, UK, 2013.

3. McNutt, J.; He, Q. Development of biolubricants from vegetable oils via chemical modification. J. Ind. Eng. Chem. 2016, 36, 1-12. [CrossRef]

4. Nagendramma, P.; Kaul, S. Development of ecofriendly/biodegradable lubricants: An overview. Renew. Sustain. Energy Rev. 2012, 16, 764-774. [CrossRef]

5. Rani, S.; Joy, M.L.; Nair, K.P. Evaluation of physiochemical and tribological properties of rice bran oil—Biodegradable and potential base stoke for industrial lubricants. Ind. Crop. Prod. 2015, 65, 328-333. [CrossRef]

6. Dalbey, W.E.; Biles, R.W. Respiratory Toxicology of Mineral Oils in Laboratory Animals. Appl. Occup. Environ. Hyg. 2003, 18, 921-929. [CrossRef]

7. Urbanus, J.H.; Lobo, R.C.; Riley, A.J. European Hazard Classification Advice for Crude Oil-Derived Lubricant Base Oils Compared with the Proposed Mineral Oil Mist TLV ${ }^{\circledR}$. Appl. Occup. Environ. Hyg. 2003, 18, 815-817. [CrossRef]

8. Dalbey, W.E.; McKee, R.H.; Goyak, K.O.; Biles, R.W.; Murray, J.; White, R. Acute, Subchronic, and Developmental Toxicological Properties of Lubricating Oil Base Stocks. Int. J. Toxicol. 2014, 33, 110-135. [CrossRef] [PubMed]

9. Wagner, H.; Luther, R.; Mang, T. Lubricant base fluids based on renewable raw materials Their catalytic manufacture and modification. Appl. Catal. A Gen. 2001, 221, 429-442. [CrossRef]

10. Yazan, D.M.; Mandras, G.; Garau, G. Environmental and economic sustainability of integrated production in bio-refineries: The thistle case in Sardinia. Renew. Energy 2017, 102, 349-360. [CrossRef]

11. Turco, R.; Pischetola, C.; Di Serio, M.; Vitiello, R.; Tesser, R.; Santacesaria, E. Selective Epoxidation of Soybean Oil in the Presence of H-Y Zeolite. Ind. Eng. Chem. Res. 2017, 56, 7930-7936. [CrossRef]

12. Turco, R.; Tesser, R.; Vitiello, R.; Russo, V.; Andini, S.; Di Serio, M. Synthesis of biolubricant basestocks from epoxidized soybean oil. Catalysts 2017, 7, 309. [CrossRef]

13. Vitiello, R.; Tesser, R.; Russo, V.; Turco, R.; Andini, S.; Di Serio, M. Loop reactor modeling for lubricants synthesis. Chem. Eng. J. 2017, 329, 295-304. [CrossRef] 
14. Vitiello, R.; Li, C.; Russo, V.; Tesser, R.; Turco, R.; Di Serio, M. Catalysis for esterification reactions: A key step in the biodiesel production from waste oils. Rend. Fis. Acc. Lincei 2017, 28, 117-123. [CrossRef]

15. Maquirriain, M.A.; Querini, C.A.; Pisarello, M.L. Glycerine esterification with free fatty acids: Homogeneous catalysis. Chem. Eng. Res. Des. 2021. [CrossRef]

16. Salimon, J.; Salih, N.; Yousif, E. Biolubricants: Raw Materials, chemical modifications and environmental benefits. Eur. J. Lipid Sci. Technol. 2010, 112, 519-530. [CrossRef]

17. Sharma, B.K.; Stipanovic, A.J. Development of a new oxidation stability test method for lubricating oils using high-pressure differential scanning calorimetry. Thermochim. Acta 2003, 402, 1-18. [CrossRef]

18. Erhan, S.Z.; Asadaukas, S. Lubricant base stocks from vegetable oils. Ind. Crop. Prod. 2000, 11, 277-282. [CrossRef]

19. Sharma, B.K.; Adhvaryu, A.; Liu, Z.; Erhan, S.Z. Chemical modification of vegetable oils for lubricant applications. J. Am. Oil Chem. Soc. 2006, 83, 129-136. [CrossRef]

20. Fernando, S.; Hanna, M.; Adhikari, S. Lubricity Characteristics of Selected Vegetable Oils, Animal Fats, and their Derivatives. Appl. Eng. Agric. 2007, 23, 5-11. [CrossRef]

21. Soni, S.; Agarwal, M. Lubricants from renewable energy sources-A review. Green Chem. Lett. Rev. 2014, 7, 359-382. [CrossRef]

22. Syahrullail, S.; Zubil, B.M.; Azwadi, C.S.N.; Ridzuan, M.J.M. Experimental evaluation of palm oil as lubricant in cold forward extrusion process. Int. J. Mech. Sci. 2011, 53, 549-555. [CrossRef]

23. Regueira, T.; Lugo, L.; Fandino, O.; Lopez, E.R.; Fernandez, J. Compressibilities and viscosities of reference and vegetable oils for their use as hydraulic fluids and lubricants. Green Chem. 2011, 13, 1293-1302. [CrossRef]

24. Siniawski, M.T.; Saniei, N.; Stoyanov, P. Influence of humidity on the tribological performance of unmodified soybean and sunflower oils. Lubr. Sci. 2011, 23, 301-311. [CrossRef]

25. Cermak, S.C.; Biresaw, G.; Isbell, T.A.; Evangelista, R.L.; Vaughn, S.F.; Murray, R. New crop oils—Properties as potential lubricants. Ind. Crop. Prod. 2013, 44, 232-239. [CrossRef]

26. Benessere, V.; Cucciolito, M.E.; Poggetto, G.D.; Di Serio, M.; Granados, M.L.; Ruffo, F.; Vitagliano, A.; Vitiello, R. Strategies for immobilizing homogeneous zinc catalysts in biodiesel production. Catal. Commun. 2014, 56, 81-85. [CrossRef]

27. Arbain, N.H.; Salimon, J. The Effects of Various Acid Catalyst on the Esterification of Jatropha Curcas Oil based Trimethylolpropane Ester as Biolubricant Base Stock. E-J. Chem. 2011, 8, 33-40. [CrossRef]

28. Oh, J.; Yang, S.; Kim, C.; Choi, I.; Kim, J.H.; Lee, H. Synthesis of biolubricants using sulfated zirconia catalysts. Appl. Catal. A Gen. 2013, 455, 164-171. [CrossRef]

29. Harmer, M.A.; Sun, Q. Solid acid catalysis using ion-exchange resins. Appl. Catal. A Gen. 2001, 221, 45-62. [CrossRef]

30. Kleinova, A.; Fodran, P.; Brncalova, L.; Cvengros, J. Substituted esters of stearic acid as potential lubricants. Biomass Bioenergy 2008, 32, 366-371. [CrossRef]

31. Aronne, A.; Di Serio, M.; Vitiello, R.; Clayden, N.J.; Minieri, L.; Imparato, C.; Piccolo, A.; Pernice, P.; Carniti, P.; Gervasini, A. An Environmentally Friendly Nb-P-Si Solid Catalyst for Acid-Demanding Reactions. J. Phys. Chem. C 2017, 121, 17378-17389. [CrossRef]

32. Russo, V.; Taddeo, F.; Cogliano, T.; Vitiello, R.; Esposito, R.; Tesser, R.; Salmi, T.; Di Serio, M. Investigation of the intrinsic reaction kinetics and the mass transfer phenomena of nonanoic acid esterification with 2-ethylhexanol promoted by sulfuric acid or Amberlite IR120. Chem. Eng. J. 2021, 408, 127236. [CrossRef]

33. Russo, V.; Rossano, C.; Salucci, E.; Tesser, R.; Salmi, T.; Di Serio, M. Intraparticle diffusion model to determine the intrinsic kinetics of ethyl levulinate synthesis promoted by Amberlyst 15. Chem. Eng. Sci. 2020, 228, 115974. [CrossRef]

34. Peters, T.A.; Benes, N.E.; Holmen, A.; Keurentjes, J.T.F. Comparison of commercial solid acid catalysts for the esterification of acetic acid with butanol. Appl. Catal. A Gen. 2006, 297, 182-188. [CrossRef]

35. Ramalingam, R.J.; Wang, M.K. Review of Recent Developments in Solid Acid, Base, and Enzyme Catalysts (Heterogeneous) for Biodiesel Production via Transesterification. Ind. Eng. Chem. Res. 2009, 48, 6162-6172. [CrossRef]

36. Kimura, M.; Nakato, T.; Okuhara, T. Water-tolerant solid acid catalysis of $\mathrm{Cs}_{2.5} \mathrm{H}_{0.5} \mathrm{PW}_{12} \mathrm{O}_{40}$ for hydrolysis of esters in the presence of excess water. Appl. Catal. A Gen. 1997, 165, 227-240. [CrossRef]

37. Turco, R.; Vitiello, R.; Tesser, R.; Vergara, A.; Andini, S.; Di Serio, M. Niobium Based Catalysts for Methyl Oleate Epoxidation Reaction. Top. Catal. 2017, 60, 1054-1061. [CrossRef]

38. Turco, R.; Di Serio, M. Sustainable synthesis of epoxidized Cynara C. Seed oil. Catalysts 2020, 10, 721. [CrossRef]

39. Hajjari, M.; Tabatabaei, M.; Aghbashlo, M.; Ghanavati, H. A review on the prospect of sustainable biodiesel production: A global scenario with an emphasis on waste-oil biodiesel utilization. Renew. Sustain. Energy Rev. 2017, 72, 445-464. [CrossRef]

40. Canakci, M.; Van Gerpen, J. Biodiesel production from oils and fats with high free fatty acids. Am. Soc. Agric. Eng. 2001, 44, 1429-1436. [CrossRef]

41. Suwannakarn, K. Biodiesel Production from High Free Fatty Acid Content Feedstocks; Clemson University, Tiger Prints: Clemson, SC, USA, 2008; p. 207.

42. Vitiello, R.; Buonerba, A.; Tesser, R.; Di Serio, M.; Grassi, A.; Santacesaria, E. Use of waste materials for biodiesel production. DGMK Tagungsbericht 2012, 3, 49-54.

43. Buonerba, A.; Cuomo, C.; Ortega Sanchez, S.; Canton, P.; Grassi, A. Gold Nanoparticles Incarcerated in Nanoporous Syndiotactic Polystyrene Matrices as New and Efficient Catalysts for Alcohol Oxidations. Chem. Eur. J. 2012, 18, 709-715. [CrossRef] 
44. Bodamer, G.W.; Kunin, R. Behavior of Ion Exchange Resins in Solvents Other Than Water-Swelling and Exchange Characteristics. Ind. Eng. Chem. 1953, 45, 2577-2580. [CrossRef]

45. ASTM D974-14e2. Standard Test Method for Acid and Base Number by Color-Indicator Titration; ASTM International: West Conshohocken, PA, USA, 2014.

46. Santacesaria, E.; Di Serio, M.; Tesser, R.; Casale, L.; Verde, D.; Turco, R.; Bertola, A. Use of a corrugated plates heat exchanger reactor for obtaining biodiesel with very high productivity. Energy Fuels 2009, 23, 5206-5212. [CrossRef]

47. Russo, V.; Protasova, L.; Turco, R.; De Croon, M.H.J.M.; Hessel, V.; Santacesaria, E. Hydrogen peroxide decomposition on manganese oxide supported catalyst: From batch reactor to continuous microreactor. Ind. Eng. Chem. Res. 2013, 52, 7668-7676. [CrossRef] 